\title{
On the Achievable Throughput in Two-Scale Wireless Networks
}

\author{
Radhika Gowaikar and Babak Hassibi \\ California Institute of Technology \\ Email: \{gowaikar, hassibi\}@ caltech.edu
}

\begin{abstract}
We propose a new model of wireless networks which we refer to as "two-scale networks." At a local scale, characterized by nodes being within a distance $r$, channel strengths are drawn independently and identically from a distance-independent distribution. At a global scale, characterized by nodes being further apart from each other than a distance $r$, channel connections are governed by a Rayleigh distribution, with the power satisfying a distance-based decay law. Thus, at a local scale, channel strengths are determined primarily by random effects such as obstacles and scatterers whereas at the global scale channel strengths depend on distance.

For such networks, we propose a hybrid communications scheme, combining elements of [8] (for distance-dependent networks) and [2] (for random networks). For a particular class of two-scale networks with $N$ nodes, we show that an aggregate throughput of the form $N^{\frac{1}{t-1}} / \log ^{2} N$ is achievable, where $t>2$ is a parameter that depends on the distribution of the connection at the local scale and is independent of the decay law that operates at a global scale. For $t<3$, this offers a significant improvement over the $O(\sqrt{N})$ results of [8].
\end{abstract}

\section{INTRODUCTION}

Sensor and ad hoc networks have seen much research activity in recent times. The first major result of the field was by Kumar and Gupta [8] where a network of $n$ nodes was studied. Strengths of the connections between two nodes were determined entirely by the distance between them and followed a deterministic power scaling law. With this model, it was shown that a throughput that scaled like $\sqrt{n}$ was the best possible. This implied that the throughput per user fell like $\frac{1}{\sqrt{n}}$ which was quite discouraging. Except when nodes were allowed to approach each other [5], similar scaling laws were shown to hold [7], [4], [6], [11], [9].

From the research on multiple antenna systems, we know that rich scattering environments, leading to independent channel coefficients between transmit and receive antennas help achieve capacity linear in the number of antennas [3], [10]. Taking cue from this, a network model with random connections was proposed in [12], [2]. In this, the channel strengths are independent of distance and geometry and are instead drawn identically and independently (i.i.d.) from a probability distribution function (p.d.f.). This model is suitable for networks over a small area, where multipath and physical obstructions dominate and the decay laws associated with farfield effects do not kick in.

While the throughput that was possible with this model depended very strongly on the distribution that the channel strengths were drawn from, several distributions, including the

\footnotetext{
${ }^{1}$ This work is supported in part by the National Science Foundation under grant nos. CCR-0133818 and CCR-0326554, by the David and Lucille Packard Foundation, and by Caltech's Lee Center for Advanced Networking.
}

Bernoulli and some heavy-tailed distributions led to throughputs that were almost linear in $n$. Thus the introduction of randomness changed the behaviour of the system significantly.

In practice, we expect neither the deterministic model of [8] nor the random model of [2] to hold. A combination of distance-dependent connections and random connections would perhaps make for a better model. In this work, we propose and analyze such a model. We assume that $N$ nodes are randomly and uniformly distributed on a sphere of radius $R$. Nodes that are within a distance $r$ from each other are connected by channels that are distance-independent. These channel strengths are assumed to be drawn i.i.d. from a distribution, $f(\cdot)$. For nodes that are further apart than $r$, the channel connections obey a Rayleigh distribution with a mean power that depends on the distance between them and follows a distance-decay law, say $g(\cdot)$.

Such a model incorporates the far field effects at a global level through the decay law, but also recognizes that obstuctions play a role at a local scale. Furthermore, appropriate choices of $r$ and $R$ can help model a full scale of networks, from the purely geometric ones of [8] to the purely random ones of [2]. A precise description of the model and the problem statement is in Section II. Sections III and IV study the scheduling and error-free communication properties of this model and the main result is stated in Section V. Examples and conclusions are presented at the end. Not surprisingly, a combination of the techniques found in [8] and [2] are employed throughout the paper.

\section{Network Model}

Consider a network with $N$ nodes that are uniformly and randomly distributed on the surface of a sphere of radius $R$. We use a sphere rather than a planar disk to separate edge effects and have symmetry between all nodes. Also, the standard convention of measuring distances along great circles will be followed.

The channel between nodes $i$ and $j$ is denoted by $h_{i, j}=$ $h_{j, i}$. Define the channel strength to be $\gamma_{i, j}=\left|h_{i, j}\right|^{2}$. The average channel strength is assumed to be distance-dependent for nodes that are more than a certain distance, say $r$, apart and independent of distance for nodes that are within a distance $r$.

More precisely, for nodes that are within a distance $r$, the channel strengths are drawn i.i.d., according to a p.d.f., say $f(\gamma)$. Let the expected value corresponding to this be denoted by $\mu_{\gamma}$.

If nodes $i$ and $j$ are at a distance $l(i, j)>r$ from each other, we model $h_{i, j}$ to be a Rayleigh distributed random variable with its power (or second moment), $E\left|h_{i, j}\right|^{2}$, given by $c g(l(i, j))$ where $g(x)$ is used to model the distancedependence and $c$ is a constant. This gives us that the corre- 
sponding $\gamma_{i, j}$ is drawn from an exponential distribution with $c g(x)$ as its mean, i.e., $c g(x) \exp (-\gamma / c g(x))$. Typically, $g(x)$ is a decreasing function such as $\frac{1}{x^{m}}$ with $m>2$ or $\frac{e^{-\delta x}}{x^{m}}$ and $c$ is chosen such that $c g(r)$ equals $\mu_{\gamma}$. This is done to ensure that the expected value of $\gamma_{i, j}$ does not change abruptly as the distance between $i$ and $j$ changes from being less than $r$ to being greater than $r$. Therefore, $c=\frac{\mu_{\gamma}}{g(r)}$.

Denote by $p_{x}(\gamma)$ the distribution from which the channel strength between two nodes with distance $x$ between them is drawn. Then we have

$$
p_{x}(\gamma)=\left\{\begin{array}{ll}
f(\gamma) & \text { if } x \leq r \\
\frac{\mu_{\gamma} g(x)}{g(r)} \exp \left(-\gamma \frac{g(r)}{\mu_{\gamma} g(x)}\right) & \text { if } x>r
\end{array} .\right.
$$

\section{A. Successful Communication}

Assume that node $i$ wishes to transmit signal $x_{i}$. We assume that $x_{i}$ is a complex Gaussian random process with zero mean and unit variance. Each node is permitted a maximum power of $P$ watts.

We incorporate interference and additive noise in our model as follows. Assume that $l$ nodes $i_{1}, i_{2}, \ldots, i_{l}$ are simultaneously transmitting signals $x_{i_{1}}, x_{i_{2}}, \ldots, x_{i_{l}}$ respectively. Suppose that node $j$ is the intended receiver of the signal $x_{i_{1}}$. Then, the signal received by node $j\left(\neq i_{1}, \ldots, i_{l}\right)$ is given by

$$
y_{j}=\sum_{t=1}^{l} \sqrt{P} h_{i_{t}, j} x_{i_{t}}+w_{j}
$$

where $w_{j}$ represents additive noise. The additive noise variables $w_{1}, \ldots, w_{N}$ are i.i.d., drawn from a complex Gaussian distribution of zero mean and variance $\sigma^{2}\left(w_{i} \sim \mathcal{C N}\left(0, \sigma^{2}\right)\right)$. The noise is statistically independent of $x_{i}$.

In equation (1), assume that only node $i_{1}$ wishes to communicate with node $j$ and the signals $x_{i_{2}}, \ldots, x_{i_{l}}$ are interference. Then the signal-to-interference-plus-noise ratio (SINR) for node $j$ is given by

$$
\rho_{j}=\frac{P \gamma_{i_{1}, j}}{\sigma^{2}+P \sum_{t=2}^{l} \gamma_{i_{t}, j}}
$$

Note that some of the interference terms will come from the exponential distribution and the others will be drawn from $f(\gamma)$, depending upon the distance of the interferer from $j$. We assume that transmission is successful when the SINR exceeds some $\rho_{0}$. If the SINR is less than $\rho_{0}$, we will say that an error has been made.

\section{B. Network Operation and Throughput}

We suppose that $K$ nodes $s_{1}, \ldots s_{K}$ are randomly chosen as sources. For every $s_{i}$, a destination node, say $d_{i}$, is chosen at random, thus making $K$ source-destination pairs. We assume that these $2 K$ nodes are all distinct and therefore $K \leq N / 2$. Source $s_{i}$ wishes to transmit message $W_{i}$ to destination $d_{i}$ and has encoded it as signal $x_{i}$.

Communications are assumed to occur using a series of hops. Every source-destination pair $\left(s_{i}, d_{i}\right)$ uses a sequence of relay nodes, each of which are expected to decode the message $x_{i}$ and retransmit it in the next time slot, using power $P$. We expect several messages to be making hops simultaneously and therefore the relay nodes have to decode in the presence of interference. With this in mind, we impose the constraint that no relay node be asked to decode two messages simultaneously. We also assume that no relay node can receive and transmit in the same time slot. These properties will define a non-colliding schedule of relaying.

Assume that all $K$ messages reach the intended destinations in (at most) $H$ time slots. Assume that a fraction $\epsilon$ of messages fail to reach the intended destination due to decoding or scheduling errors. Each message contains at least $\log \left(1+\rho_{0}\right)$ bits of information since $\rho_{0}$ is the SINR threshold. Therefore, we define the throughput as

$$
T=(1-\epsilon) \frac{K}{H} \log \left(1+\rho_{0}\right)
$$

Note that all the quantities above may depend on $N$. Typically, we force $\epsilon$ to go to zero. In the rest of this paper, we present a scheme of scheduling and communicating and analyze the throughput as well as performance of this scheme. Our concern will primarily be with arbitrarily large values of $N$. Thus, we will obtain an asymptotic achievability result for the throughput $T$.

\section{RELAYING SCHEME}

In this section we determine the scheduling of the relay nodes for the multihop protocol. We do this through various constructions, including Voronoi tessellations, a superschedule and many subschedules. We will borrow techniques from [8] and [2] and put them together in a suitable manner to perform scheduling for the proposed hybrid model.

\section{A. Tessellations and cell-aggregates}

Recall the concept of a Voronoi tessellation, used extensively in [8]. Lemma 4.1 of [8] establishes the existence of a Voronoi tessellation of the surface of the unit sphere where each Voronoi cell contains a disk of radius $\delta$ and is contained in a disk of radius $2 \delta$ for any $\delta>0$. We will use this result for the surface of the sphere of radius $R$. (This can be done by using the original result for $\delta / R$ rather than $\delta$ and then scaling the obtained tessellation by a factor of $R$.) Denote by $\mathcal{T}(x)$ a tessellation of the surface of the sphere of radius $R$ where each Voronoi cell contains a disk of radius $x$ and is contained in a disc of radius $2 x$. In particular, consider a tessellation $\mathcal{T}(r / 12)$ where $r$ is the radius within which channel strengths are distance-independent and are drawn i.i.d. from $f(\gamma)$. Cells of this tessellation will be labelled $S_{i}$. It is easy to show that in such a tessellation, for any cell, $S_{i}$, it and all its neighboring cells are contained in a disk of diameter $r$. (A similar, though slightly different, result is shown in Lemma 4.2 of [8].) Thus, every connection within this set of cells is drawn i.i.d. according to $f(\gamma)$. Recall that the area of a circle of radius $x$ on the surface of a sphere of radius $R$ is given by $A(x)=4 \pi R^{2} \sin ^{2} \frac{x}{2 R}$. Using this fact, it is possible to show that the number of cells that are neighbors to a given cell is bounded by a constant, say $c_{1}$.

\section{B. Determining a Superschedule}

Assume that such a tessellation of the surface of the sphere is done once and fixed. We refer to this as $\mathcal{T}_{0}(r / 12)$. Every node belongs to some $S_{i}$. (Nodes lying on cell boundaries can be assigned arbitrarily.) Consider the source-destination pair $\left(s_{i}, d_{i}\right)$. Denote by $L_{i}$ the line segment connecting them. This segment passes through several cells in order as it traverses from $s_{i}$ to $d_{i}$. Note that the maximum number of cells it can pass through is $M=c_{2} \frac{R}{r}$ for some constant $c_{2}$. Denote these cells, in sequence, by $s_{i} \in S_{i, 0}, S_{i, 1}, S_{i, 2}, \ldots, S_{i, M} \ni d_{i}$. 
(Some sequences may, in actuality, be shorter than $M$.) We will refer to the set of cells $S_{1, t}, S_{2, t}, \ldots, S_{K, t}$ as the $t$-th layer of cells.

The schedule described above only tells us the cells that a particular message has to pass through in a particular layer. We now decide which node in a particular cell is responsible for which message in a particular layer of transmission.

There are at least $\frac{4 \pi R^{2}}{A(r / 6)}=1 / \sin ^{2} \frac{r}{12 R}$ cells in $\mathcal{T}_{0}(r / 12)$. The $K$ sources are assumed to be uniformly distributed on the surface of the sphere. Therefore, each cell has at most $K \sin ^{2} \frac{r}{12 R}=k_{1}$ sources. (This can be made more rigorous.) Thus, that cell occurs in the zeroth layer around $k_{1}$ times. In general, a cell occurs in the $t$-th layer around $k_{1}$ times.

We will require that the $K$ nodes that act as relay nodes in one layer be distinct from each other as well as the $K$ nodes occuring in the previous layer. This is equivalent to requiring the $k_{1}$ relay nodes in each cell of the $t$-th layer to be distinct from each other as well as the $k_{1}$ nodes from the same cell occuring in the previous layer. In the zeroth layer of transmission, this condition is trivially met since the $K$ distinct original source nodes (around $k_{1}$ of them occuring in each cell) start out having the messages that need to be relayed and there is no previous layer. We wish to have such distinct nodes for the $i$-th layer assuming that such nodes for each layer upto the $(i-1)$-th have already been determined. Let us determine the conditions under which this is possible.

Consider a specific cell in $\mathcal{T}_{0}(r / 12)$. This is expected to have $k_{1}$ distinct nodes that are the chosen relays in the $(i-1)$ th layer. This cell also occurs $k_{1}$ times in the $i$-th layer and we wish to assign a further $k_{1}$ distinct relay nodes for each occurence. The total number of nodes in this cell is at least $N /(\#$ maximum number of cells $)=N /\left(4 \pi R^{2} / A(r / 12)\right)=$ $N \sin ^{2} \frac{r}{24 R}=n_{1}$. Therefore our condition of distinct nodes can be met if $2 k_{1} \leq n_{1}$. After simplification, this gives the condition

$$
K \leq N /\left(8 \cos ^{2} \frac{r}{24 R}\right)
$$

Once this condition is satisfied, we can assign a distinct relay node for each of the $K$ messages in each layer. The relay node in layer $t$ that is responsible for message $i$ will be called $s_{i, t}$. (Clearly, $s_{i, t} \in S_{i, t}$.) We refer to the $K$ sequences $s_{i}=s_{i, 0}, s_{i, 1}, \ldots, s_{i, M}=d_{i}$ for $i=1, \ldots, K$ as the superschedule. It now remains to decide how to route the message $i$ from its relay node in layer $t$, namely $s_{i, t}$ to its relay node in layer $(t+1)$, namely, $s_{i, t+1}$. We refer to this as subscheduling and address it next.

\section{Non-colliding Subschedules}

We will consider time slots in blocks of size $h$, where $h$ denotes the (maximum) number of hops required for a message to be transmitted from $s_{i, j}$ to $s_{i, j+1}$. In a specific block of time slots, say from $v h+1$ to $(v+1) h$, some constant fraction $c_{3}$ of all cells will be chosen at random and called active cells. We choose $c_{3}<1 / c_{1}$ where $c_{1}$ is an upper bound on the number of neighbors of a cell. Denote the set of chosen cells by $T_{v}$. Consider the cells that are not in $T_{v}$. Let $j$ be such a cell. If one of the neighbors of $j$ is in $T_{v}$, assign $j$ to it. If more than one of the neighbors of $j$ are in $T_{v}$, this assignment can be done randomly. Thus, for each of the $\left|T_{v}\right|$ originally chosen cells, we now have $\left|T_{v}\right|$ cell-aggregates that are active. (Some of these may consist of just one cell, namely, the originally chosen cell.) Figure 1 demonstrates this. In the $v$-th block of time slots, communications will occur only within the $T_{v}$ cell aggregates and not across one aggregate to another. Since any cell and its neighbors can be put inside a circle of diameter $r$, connections with an aggregate are drawn i.i.d. from $f(\gamma)$. We will make use of this fact in determining $h$ and a non-colliding subschedule in Lemma 1.

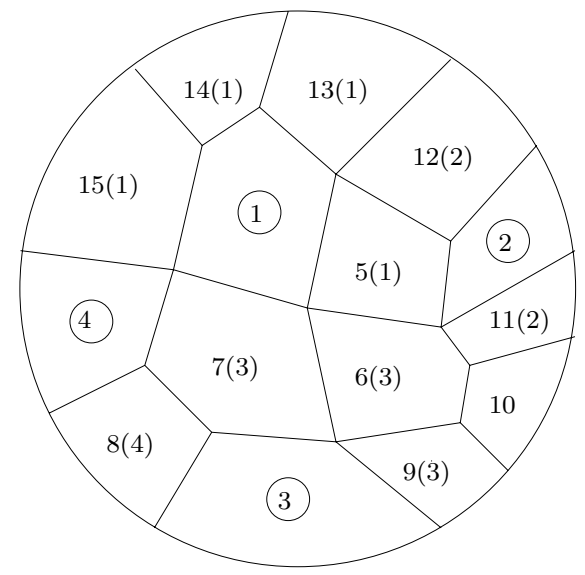

Fig. 1. Cells 1, 2, 3, 4 (circled) are originally chosen to be in $T_{v}$. The remaining cells are then assigned as indicated in parantheses. For example 13 gets assigned to 1 and 6 to 3 . Cell 10 remains unassigned. The aggregate corresponding to cell 3 consists of cells 3, 6, 7 and 9 .

A particular choice of $T_{v}$ leads to some pairs of adjacent cells not being in the same cell-aggregate. For a pair that gets split into two cell-aggregates, the relays in one cell that have the next relay in the other cell are unable to communicate with each other in the $v$-th block of time slots. However, there is a probability that in another set, say $T_{w}$, this pair does not get split up. Let $B$ be the number of sets we have to choose in order for every pair of adjacent cells to have been chosen in the same aggregate at least once.

Let $i$ and $j$ be adjacent cells. They can be in the same cellaggregate in a randomly obtained $T_{v}$ if $\left(i \in T_{v}, j \notin T_{v}\right.$ and $j$ gets assigned to $i$ ) or vice versa. By symmetry, both cases are equally likely. Therefore,

$\mathrm{P}(i, j$ are in the same cell-aggregate $)$

$=2 \mathrm{P}\left(i \in T_{v}, j \notin T_{v}, j\right.$ gets assigned to $\left.i\right)$

$=2 \mathrm{P}\left(i \in T_{v}\right) \mathrm{P}\left(j \notin T_{v} \mid i \in T_{v}\right) \mathrm{P}\left(j\right.$ is assigned to $\left.i \mid i \in T_{v}, j \notin T_{v}\right)$

$\geq 2 c_{3}\left(1-c_{3}\right) \frac{1}{c_{1}}$

The last expression comes from the fact that a fraction $c_{3}$ of cells are chosen at random to be in $T_{v}$. Therefore $i$ is in $T_{v}$ with probability $c_{3}$ and $j$ is not in $T_{v}$ with probability $\left(1-c_{3}\right)$ independently of $i$. Finally, $j$ has at most $c_{1}$ neighbors, including $i$. If some $x$ of them are chosen in $T_{v}$ (and $i$ is one of them), the probability of $j$ being assigned to $i$ is $1 / x \geq 1 / c_{1}$.

Let $c_{4}=2 c_{3}\left(1-c_{3}\right) \frac{1}{c_{1}}$. Any choice of $c_{3}<c_{1} / 2$ ensures that $c_{4}<1$. Therefore, the probability that $i$ and $j$ are not in the same cell-aggregate in $B$ choices for sets of cell-aggregates is bounded above by $\left(1-c_{4}\right)^{B}=e^{B \log \left(1-c_{4}\right)}$. If we choose $B$ to be $\log N$, this behaves as $N^{\log \left(1-c_{4}\right)}$ which goes to zero as $N$ goes to infinity. (It is clear that $B$ can be chosen to be any function that goes to infinity for large $N$.)

Consider a block in which a particular cell-aggregate is active. Assume that it consists of $c_{5} \leq 1+c_{1}$ cells. Each 
cell has around $k_{1}$ relays that wish to transmit and $k_{1}$ relays that wish to receive in a particular layer. Thus, we expect there to be no more than $c_{5} k_{1}$ transmissions that need to take place while that cell-aggregate is active. We denote the actual number of transmissions by $k$. In addition, the cellaggregate lies entirely in a circle of diameter $r$, therefore all the connection strengths within it are drawn i.i.d. from the distribution $f(\gamma)$. Let $n=c_{5} n_{1}$ be the total number of nodes in the aggregate.

In this subnetwork of $n$ nodes with i.i.d. connections we seek a schedule of $k$ non-colliding paths from the set of transmitting relays to the set of receiving relays. But this is exactly the problem that is addressed in [2].

\section{Good edges and vertex-disjoint paths}

We reproduce the solution presented there. The channels that are stronger than a chosen parameter $\beta$ are called good. All communications take place over good channels. Since channels are drawn i.i.d. from $f(\gamma)$, for every channel, there is a probability $p=P(\gamma \geq \beta)$ of its being good. We now construct a graph on $n$ vertices where each vertex represents a node of the network. An edge is drawn between two vertices if the channel between the corresponding nodes is good. Thus, we obtain a graph on $n$ vertices where edges are drawn i.i.d. from a Bernoulli distribution of parameter $p$.

Such a graph fits a standard random graph model called $\mathcal{G}(n, p)$. This model is well-studied and we appeal to an existing result in the literature to help us with our scheduling. We seek $k$ non-colliding paths that go from the set of $t$-th layer relay nodes to the respective $(t+1)$-th layer relay nodes. In [1], an identical problem is studied, but the condition on the paths is stricter still - no two paths can share a vertex. In other words, the paths must be vertex-disjoint. We state here the result of [1] as it applies to our problem.

Lemma 1: Suppose that $G=G(n, p)$ and $p \geq \frac{\log n+\omega_{n}}{n}$, where $\omega_{n} \rightarrow \infty$. Then there exists a constant $\alpha>0$ such that, with probability approaching 1 , there are vertex-disjoint paths connecting $x_{i}$ to $y_{i}$ for any set of disjoint, randomly chosen node pairs

$$
F=\left\{\left(x_{i}, y_{i}\right) \mid x_{i}, y_{i} \in\{1, \ldots, n\}, i=1, \ldots, k\right\}
$$

provided $k=|F|$ is not greater than $\alpha n \frac{\log n p}{\log n}$.

The $x_{i} \mathrm{~s}$ of the result above are the transmitting relays (from the $t$-th layer) and the $y_{i} \mathrm{~s}$ are the corresponding receiving relays (from the $(t+1)$-layer). From Section III-B, we know that these are all distinct nodes. We have $k=c_{5} k_{1}=$ $c_{5} K \sin ^{2} \frac{r}{12 R}$ and $n=c_{5} N \sin ^{2} \frac{r}{24 R}$. Therefore the above theorem establishes the existence of the required non-colliding paths if $c_{5} K \sin ^{2} \frac{r}{12 R} \leq \alpha c_{5}\left(N \sin ^{2} \frac{r}{24 R}\right) \frac{\log c_{5} N \sin ^{2} \frac{r}{24 R} p}{\log c_{5} N \sin ^{2} \frac{r}{24 R}}$ or

$$
K \leq \alpha N \frac{\log c_{5} N \sin ^{2} \frac{r}{24 R} p}{\log c_{5} N \sin ^{2} \frac{r}{24 R}} \frac{1}{\cos ^{2} \frac{r}{24 R}} .
$$

Recall that for every block of $h$ time slots, we have certain active cell-aggregates. Each time a cell-aggregate is active, we can appeal to the above theorem to get a satisfactory schedule. Additionally, it is possible to show that the lengths of the vertex-disjoint paths grow no faster than $\frac{\log n}{\alpha \log n p}$. Therefore, the time slots required, $h$, are bounded above by $h \leq \frac{\log n}{\alpha \log n p}=\frac{\log c_{4} N \sin ^{2} \frac{r}{24 R}}{\alpha \log c_{4} N \sin ^{2} \frac{r}{24 R} p}$.
Putting the results of this section together, we have the following result.

Theorem 1: All $K$ communications can be scheduled in $H=h M B=\frac{\log n}{\alpha \log n p} \cdot c_{2} \frac{R}{r} \cdot \log N$ time slots using noncolliding paths of length $h M=\frac{\log n}{\alpha \log n p} \cdot c_{2} \frac{R}{r}$ provided the following conditions hold.

1) $K \leq N /\left(8 \cos ^{2} \frac{r}{24 R}\right)$.

2) $K \leq \alpha N \log n p /\left(\log n \cdot \cos ^{2} \frac{r}{24 R}\right)$.

Here, $\frac{\log n+\omega_{n}}{n} \leq p \leq 1$ is a probability, $n=c_{5} N \sin ^{2} \frac{r}{24 R}$, $\alpha$ and $c_{5}$ are constants, and $\omega_{n}$ can be any function that goes to infinity.

Thus, the hybrid model allows us to schedule non-colliding paths using a combination of ideas from the deterministic model of [8] and the random model of [2]. The next question to investigate is that of an appropriate SINR threshold, $\rho_{0}$ that determines the rate of the transmissions.

\section{PRobABILITy of ERror}

All transmissions take place in the presence of noise and interference. The SINR threshold $\rho_{0}$ has to be carefully set so that it is not too low, but is low enough to ensure that most communications are successful. Let us investigate the SINR at any particular hop. Let us assume that node $a$ is transmitting to node $b$. The power of the transmission is $P$. All communications take place on channels that are good, that is, where $\gamma \geq \beta$. Therefore, the signal power is at least $P \beta$. The additive noise power is $\sigma^{2}$. There is interference from all other transmissions that occur in the same time slot. Some of these transmitting nodes lie within a distance $r$ of the receiving node $b$ and others lie further.

Consider the interferers lying within a distance $r$. There are around $k_{2}=K \frac{A(r / 2)}{4 \pi R^{2}}=K \sin ^{2} \frac{r}{4 R}$ of them, say $u_{1}, \ldots, u_{k_{2}}$ and the interference from them is given by

$$
I_{\text {inside }}=P \sum_{i=1}^{k_{2}} \gamma_{u_{i}, b}
$$

The expected value of this is easily calculated and $E I_{\text {inside }}=$ $P k_{2} \mu_{\gamma}=P K \sin ^{2} \frac{r}{2 R} \mu_{\gamma}$.

The other interferers lie further than a distance $r$ from the $b$. Let us assume that there are $K$ such interferers. (This in an overestimate since we have $K$ paths in total and do not expect them all to be active at the same time.) Therefore, the total interference from them is given by

$$
I_{\text {outside }}=P \sum_{i=1}^{K} \gamma_{u_{i}, b}
$$

where the $\gamma_{u_{i}, b}$ are exponential random variables with mean $\frac{\mu_{\gamma} g\left(l\left(u_{i}, b\right)\right)}{g(r)}$ and $l\left(u_{i}, b\right)$ is the distance between $u_{i}$ and $b$.

We now calculate the expected value of $I_{\text {outside. }}$ Let us represent the density of these interferers by $\kappa=K / 4 \pi R^{2}$. Consider an infinitesimally thin annulus of radius $t>r$ and width $d t$ centered at $b$. Since we are on the sphere, the area of this annulus is less than $2 \pi t d t$ and the number of interferers in this annulus is $\kappa 2 \pi t d t$. In the expression for $I_{\text {outside }}$ above, there are around these many terms with mean $\frac{\mu_{\gamma} g(t)}{g(r)}$. Therefore we have

$$
E I_{\text {outside }} \leq \int_{r}^{\infty} P \kappa 2 \pi t \frac{\mu_{\gamma} g(t)}{g(r)} d t=P K \frac{r^{2} \mu_{\gamma}}{2 R^{2}}
$$


in the case where $g(t)=1 / t^{m}, m>2$.

We have this bound on the SINR for node $b$.

$$
\rho_{b} \geq \frac{P \beta}{\sigma^{2}+I_{\text {inside }}+I_{\text {outside }}} .
$$

The probability that the SINR falls below some threshold $\rho_{0}$ is bounded as follows.

$$
\begin{aligned}
\mathrm{P}\left(\rho_{b} \leq \rho_{0}\right) & \leq \mathrm{P}\left(\frac{P \beta}{\sigma^{2}+I_{\text {inside }}+I_{\text {outside }}} \leq \rho_{0}\right) \\
& =\mathrm{P}\left(I_{\text {inside }}+I_{\text {outside }} \geq \frac{P \beta}{\rho_{0}}-\sigma^{2}\right) \\
& \leq \frac{E\left(I_{\text {inside }}+I_{\text {outside }}\right)}{\frac{P \beta}{\rho_{0}}-\sigma^{2}} \leq \frac{K \sin ^{2} \frac{r}{4 R} \mu_{\gamma}+K \frac{r^{2} \mu_{\gamma}}{2 R^{2}(3)}}{\frac{\beta}{\rho_{0}}-\frac{\sigma^{2}}{P}}
\end{aligned}
$$

where the Markov inequality and the expected values of the interferences have been used in the last line.

We will set the SINR threshold to

$$
\rho_{0}=\frac{P \beta}{\sigma^{2}+a\left(P k \mu_{\gamma}+P K \frac{r^{2} \mu_{\gamma}}{2 R^{2}}\right)}
$$

where $a \geq 1$ can be suitably chosen to make transmissions error free. This value of $\rho_{0}$ is chosen keeping in mind that the interference terms are expected to behave like their expected values for large networks. We use $a$ to keep the threshold conservative.

Finally, we know that every message makes $h M=$ $\frac{\log n}{\alpha \log n p} c_{2} \frac{R}{r}$ hops as described in Section III. At each hop, the probability that the SINR falls below the threshold $\rho_{0}$ is as calculated above. With a simple union bound, it is possible to show that a message fails to reach its destination with probability $\epsilon$ where

$$
\epsilon \leq \# \text { hops } \cdot P\left(\rho_{b} \leq \rho_{0}\right) \leq \frac{\log n}{\alpha \log n p} c_{2} \frac{R}{r} \frac{1}{a}
$$

The value of $\rho_{0}$ as given in (4) and the expression of (3) have been used.

\section{Deriving the Main Result}

We now have all the pieces we need to obtain the final result. Section III tells us the conditions for the existence of a non-colliding schedule and Section IV tells us the conditions for communications to be successful with this schedule. We thus have the following result.

Theorem 2: Consider a network of $N$ nodes, uniformly and randomly distributed over the surface of a sphere of radius $R$. For two nodes within a distance $r$, channel strengths are drawn i.i.d. from a pdf $f(\gamma)$ with mean $\mu_{\gamma}$. Otherwise they are drawn from an exponential distribution with a mean of $\mu_{\gamma} r^{m} / x^{m}$, where $x>r$ is the distance between them. Let $F(\gamma)$ denote the cumulative distribution function of $f(\gamma)$ and $Q(\gamma)=1-F(\gamma)$. Let $n=c_{5} N \sin ^{2} \frac{r}{24 R}$ where $c_{5}$ is a known constant. Choose any $\beta$ such that $p=Q(\beta)=\frac{\log n+\omega_{n}}{n}$, where $\omega_{n} \rightarrow \infty$ as $n \rightarrow \infty$. Let $A(x)=4 \pi R^{2} \sin ^{2} \frac{x}{2 R}$. Then a throughput of

$T=(1-\epsilon) \frac{\alpha K r \log n p \cdot \log \left(1+\frac{P \beta}{\sigma^{2}+a\left(P K \sin ^{2} \frac{r}{4 R} \mu_{\gamma}+P K \frac{r^{2} \mu_{\gamma}}{2 R^{2}}\right)}\right)}{\log n \cdot c_{2} R \cdot \log N}$

is achievable where $\alpha$ and $c_{2}$ are constants and $K$ and $a \geq 1$ are chosen such that the following conditions are satisfied.
1) $K \leq N /\left(8 \cos ^{2} \frac{r}{24 R}\right)$.

2) $K \leq \alpha N \log n p /\left(\log n \cdot \cos ^{2} \frac{r}{24 R}\right)$.

3) $\epsilon \leq \frac{\log n}{\alpha \log n p} \cdot \frac{R}{r} \cdot \frac{1}{a} \rightarrow 0$

Proof: From theorem 1 we know the number of hops required for a non-colliding schedule and the necessary conditions. From (4) and (5) in Section IV we know $\rho_{0}$ and the condition for successful communications. Recalling that the throughput is $T=(1-\epsilon) \frac{K}{H} \log \left(1+\rho_{0}\right)$, we get the above theorem.

\section{EXAMPLE AND CONCLUSION}

Consider $f(\gamma)=\frac{1}{(1+\gamma)^{t}}$ with $t>2$ as the distribution from which the channel strengths are drawn i.i.d. for nodes within a distance $r$ from each other. We need $t>2$ for $\mu_{\gamma}$ to be finite. We will assume that the other connections are exponential with the mean following a distance decay law of $g(x)=1 / x^{m}$ for $m>2$. Choosing $p=\frac{2 \log n}{n}$, we get a $\beta$ that behaves like $(n(t-1) / 2 \log n)^{\frac{1}{t-1}}-1$. Since $r$ and $R$ are fixed, we can approximate $\sin ^{2} \frac{r}{4 R}$ with $c_{6} \frac{r^{2}}{R^{2}}$. Therefore, $n=c_{5} c_{6} N \frac{r^{2}}{R^{2}}=c_{7} N$ and $\beta \approx N^{\frac{1}{t-1}} / \log \frac{1}{t-1} N$. One can choose $K$ to be of the form $N / \log N$ and $a$ of the form $\log \log N \frac{\log n}{\alpha \log n p} \frac{R}{r}$. This satisfies the required conditions of the theorem and we get a throughput of $T=N^{\frac{1}{t-1}} / \log ^{2} N$. For $t$ just greater than 2 , this is almost linear but for $t>3$, it falls below $\sqrt{N}$. It is interesting to note that $m$ plays no role in this analysis.

To conclude, we have proposed a two-scale network model in which local connections are drawn at random and global connections depend on a distance-based decay law. We have analyzed the throughput for this network and found that depending on the chosen parameters it can give a wide range of throughputs.

\section{REFERENCES}

[1] A. Z. Broder, A. M Frieze, S. Suen, E. Upfal, "An Efficient Algorithm for the Vertex-Disjoint Paths Problem in Random Graphs," Proc 7th Symp. Discrete Algorithms, Atlanta, 1996, pp 261- 268.

[2] R. Gowaikar, B. Hochwald, B. Hassibi, "Communication over a Wireless Network with Random Connections," IEEE Transactions on Information Theory, July 2006

[3] G. J. Foschini, "Layered space-time architecture for wireless communication in a fading environment when using multi-element antennas," Bell Labs. Tech. J., vol. 1, no. 2, pp. 41-59, 1996.

[4] M. Gastpar and M. Vetterli, "On the capacity of wireless networks: the relay case," Proc. 21st INFOCOM, New York, Jun. 2002, pp. 1577-1586.

[5] M. Grossglauser and D. Tse, "Mobility increases the capacity of ad-hoc wireless networks," IEEE/ACM Trans. on Networking, vol. 10, pp. 477486, Aug. 2002.

[6] O. Léveque and E. Telatar, "Information theoretic upper bounds on the capacity of large extended ad hoc wireless networks," to appear in the IEEE Trans. on Info. Theory.

[7] M. Franceschetti, O. Dousse, D. Tse and P. Thiran, "On the throughput capacity of random wireless networks," submitted to IEEE Trans. Info. Theory, 2004

[8] P. Gupta and P. R. Kumar "The capacity of wireless networks," IEEE Trans. Info. Theory, vol. 46, pp. 388-404, Mar. 2000.

[9] P. Gupta and P. R. Kumar, "Towards an information theory of large networks: an achievable rate region," IEEE Trans. Info. Theory, vol. 49, pp. 1877-1894, Aug. 2003.

[10] I. E. Telatar, "Capacity of multi-antenna Gaussian channels," Eur. Trans. Telecom., vol. 10, pp. 585-595, Nov. 1999.

[11] L.-L. Xie and P. R. Kumar, "A network information theory for wireless communication: Scaling laws and optimal operation," IEEE Trans. Info. Theory, vol. 50, pp. 748-767, May 2004.

[12] R. Gowaikar, B. Hochwald, B. Hassibi, "An Achievability Result for Random Networks," Proc. IEEE ISIT 2005, Adelaide, Australia, pp 946-
950. 